\title{
Courting controversy online
}

\section{Carla Figueira de Morisson \\ Faria and Andrew Brown say \\ that academic debate can still be \\ fostered in an online-only world}

The past six months have seen scientists shift from working in the lab to conducting their research and collaborations online using tools such as Zoom. Conferences, which had almost always been held inperson before the COVID-19 pandemic, have also had to switch to online-only. This move has led some to warn of the long-term dangers for science, especially those fields in which there is "much disagreement and passion". Face-to-face meetings, they contend, are the "only way to propel science forward" (see July p19).

We disagree. We recently co-organized an online conference devoted entirely to controversy. Held in early July over three days, the Quantum Battles in Attoscience event had more than 300 registered participants from 34 countries. Attoscience is a fairly new branch of physics and deals with some of the shortest times in nature $\left(10^{-18} \mathrm{~s}\right)$. At these time scales, researchers can image the real-time movements of electrons. And since electrons carry energy in systems from biomolecules to nanostructures and metals, attoscience may impact many areas of science and even lead to "optoelectronic" computers.

Despite - or perhaps because of - its vibrancy, the attoscience community is very divided on almost all issues, siloed into factions without a co-ordinated effort toward constructive debate. We have seen plenty of "street fights" at major international conferences and in journals, with not much respect being held between the different parties. The idea of embracing, instead of avoiding, conflict emerged when we were writing a workshop proposal. Privately, some of us had practised martial arts, where rigorous codes of conduct are enforced. Breaking them will result in your being expelled for tarnishing your school's reputation. In extreme cases, you may even have your belts revoked. This approach is very different from academic street fights, so we asked: "If people want to fight, why not go for the scientific equivalent of a martial-arts tournament?"

\section{Mortal combat}

We initially intended to host a "battle" event at University College London, but moved it online when the pandemic hit.

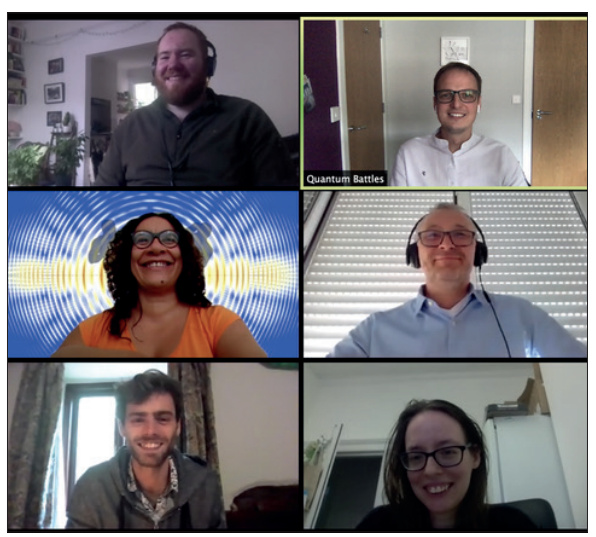

surance that they would not be caught off guard. The preparation allowed them to explore controversial points, let go of their impostor syndrome and step outside their comfort zones to discuss more "fringe" topics. The mock battles helped to set boundaries and timing, ensuring that everyone was equally represented. The battles were mediated by leading scientists in the field who were not affiliated with the panelists. They met a few times to establish how the battles would be conducted.

Our conference still boasted big names but they were invited to give more traditional talks. By focusing on early-career researchers we could avoid a lot of politics and ego: the combatants were willing to invest in the process precisely because they had more to gain from it. During the conference, it was also much easier to poll people online as anonymity helped to increase audience participation. In a real conference we would only have the usual suspects asking or replying to questions.

\section{why not go for the \\ Culture change} scientific equivalent

\section{of a martial-arts}

\section{tournament?}

This posed several challenges, but also gave us plenty of opportunity to test this debating format with a specific code of conduct that was developed especially for the event. We invited early-career researchers - who we dubbed "combatants" - from opposing groups to participate in three "battles" on contentious topics. Every combatant was promoted on the workshop website and on social-media platforms leading up to the conference. They also became co-organizers of the conference, invested enormously in the planning of the battles and passed on the excitement to their groups.

Bringing these people together to trust each other in a virtual environment took around three months. This was done via Zoom meetings and dedicated channels on Slack. Two organizers - Bridgette Cooper and Andrew Maxwell - managed the interaction between participants. Once the arguments had been agreed and prepared, we then carried out several mock battles. Traditionally, panel discussions happen on the fly and involve leaders in the field, who would not have the time for such a lengthy preparation. We heard from our combatants that as early-career researchers they wanted the practice as well as the reas-
Current conference culture is built to encourage the participation of principal investigators. This needs to change - why do we need the same lectures every year from the same people? We would like to see fresh faces and ideas, but this is a double-edged sword: a conference with no big names may not attract interest and may even look suspicious. With this new initiative we wanted to change this mindset. Furthermore, an onsite conference requires a huge investment in terms of local resource, sponsorship and infrastructure, both for the participants and the organizers. This poses further barriers and favours those with privilege and time. Online meetings avoid some of these issues.

The meeting was a huge success and by subverting a few paradigms, we hope to have shown that alternatives are possible. Not only can debate happen in an online forum, but it can be done while maintaining respect for those involved.

- www.quantumbattles.com
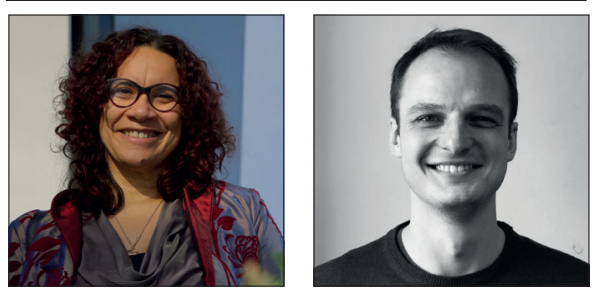

Carla Figueira de Morisson Faria is at University College London and Andrew Brown is at Queen's University Belfast, e-mail c.faria@ucl.ac.uk 